\title{
The diathermy scratch pad: A cheap and efficient tool for chemical and explosion-related burns
}

\author{
Allen Wei-Jiat Wong ${ }^{1}$, Qi En Hong ${ }^{1}$, Cheryl Li Yu Hui ${ }^{1}$, Si Jack Chong ${ }^{2}$ \\ ${ }^{I}$ Department of Plastic, Reconstructive and Aesthetic Surgery, Singapore General Hospital, Singapore; ${ }^{2}$ Plastic, Reconstructive and Aesthetic \\ Surgery Service, Sengkang General Hospital, Singapore
}

The burn center in our hospital is a national and regional (Southeast Asia) center. Of all admissions, 10\% are related to blast explosions, and $8 \%$ due to chemical burns. In the acute burn management protocol of Singapore General Hospital, early surgical debridement is advocated for all acute partial-thickness burns. The aim of early surgical debridement is to remove all debris and unhealthy tissue, preventing wound infection and thereby expediting wound healing. In chemical burns, there can be stubborn eschars that are resistant to traditional debridement. We would like to present a novel technique using the diathermy scratch pad as a cheap and efficient tool for the dual purpose of surgical debridement and dermabrasion.

Keywords Burns / Foreign bodies / Explosion / Dermabrasion / Debridement
Correspondence: Allen Wei-Jiat Wong Department of Plastic, Reconstructive and Aesthetic Surgery, Singapore General Hospital, Outram Road, Singapore 169608, Singapore

Tel: $+65-6321-4686$

Fax: $+65-6220-9340$

E-mail: allen.wong.wei.jiat@gmail.com

Received: 10 May 2018 • Revised: 8 Jul 2018 • Accepted: 22 Oct 2018

pISSN: 2234-6163 • elSSN: 2234-6171 • https://doi.org/10.5999/aps.2018.00507• Arch Plast Surg 2019;46:88-91

\section{INTRODUCTION}

The burn center in Singapore General Hospital is a national and regional (Southeast Asia) center [1,2]. Of all admissions, $10 \%$ are related to blast explosions, and $8 \%$ due to chemical burns [1]. In our hospital's acute burn management protocol, early surgical debridement is advocated for all acute partial-thickness burns $[3,4]$. The aim of early surgical debridement is to remove all debris and unhealthy tissue, preventing wound infection and thereby expediting wound healing. Early surgical debridement in cases of chemical burns also allows us to physically remove chemical-laden debris that has only been partially neutralized, therefore preventing further burn conversion. In explosion-related burns, foreign body tattooing is common, and dermabrasion is an effective way of treating large affected areas. We would like to present a novel technique that uses the diathermy scratch pad (Fig. 1) as a cheap and efficient tool for the dual purpose of surgical debridement and dermabrasion in partial-thickness burns, especially when explosions and chemicals are involved.

\section{IDEAS}

The technique that we describe here is useful for burns related to chemicals and explosions. Prior to debridement, the depth of the burns needs to be accurately accessed. Mid-dermal partialthickness burns are the deepest burns to which this technique can be applied. For burns deeper than this level, tangential excision is recommended instead.

\section{Chemical burns}

Acid burns cause coagulative necrosis. Upon contact with skin, coagulative necrosis occurs on the epidermis, forming a very adherent hyperpigmented eschar that is difficult to remove. The diathermy scratch pad is very effective for this type of burnt skin 
(Figs. 2 and 3).

Under general anesthesia, the skin is cleansed with $10 \%$ povidone-iodine solution. Irrigation with saline solution is performed, using universal $\mathrm{pH}$ indicator strips as a guide to the neutralization endpoint. Complete neutralization may not be achievable because the chemical may have already penetrated the tissue, and such areas can only be removed through surgical debridement. Once the best achievable $\mathrm{pH}$ has been reached, the surgeon can then proceed to surgical debridement using a Valleylab (Covidien, Dublin, Ireland) diathermy scratch pad. To facilitate debridement, the self-adhesive of the pad can be attached to the finger-tips of the middle three fingers or the thumb. First, by using a rhythmic sideways sweeping motion of the wrist, the surgeon can gently debride the affected areas. Second, by varying the strength of pressure, the surgeon can adjust the depth of debridement. Third, if precision is required, the

\section{Fig. 1. Diathermy scratch pad}

The Valleylab diathermy scratch pad comes with a self-adhesive back and is sterile.

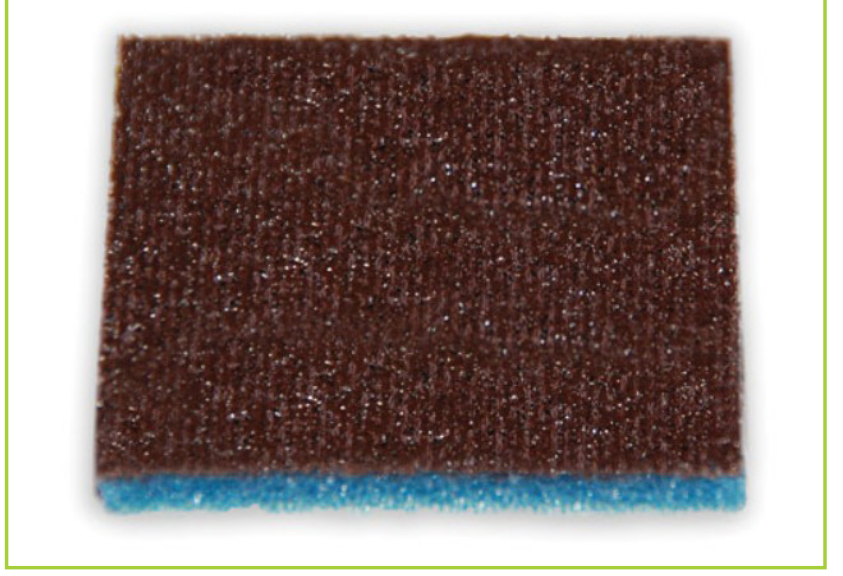

surgeon can fold the diathermy scratch pad around the index finger, and gently rub the region in small circular motions, as shown in the Supplemental Video 1. Using variations in angle, pressure and speed, the surgeon can tailor the debridement to the requirements of the wound. Debridement with the diathermy scratch pad is performed till healthy dermis is encountered. The wound is further irrigated with saline and checked with a universal $\mathrm{pH}$ indicator strip. The chemical should be totally neutralized and the universal $\mathrm{pH}$ indicator strip should show a slightly acidic physiological $\mathrm{pH}$ of about 7.35. Biobrane can now be applied as a wound dressing, with iodine gauze as secondary dressing as per our protocol $[3,4]$.

\section{Explosion-related burns}

In cases of explosion-related burns, in addition to thermal burns, patients also suffer penetrating injuries from shrapnel. Foreign body tattooing can occur from smaller pieces of shrapnel, which can be efficiently removed with dermabrasion. The diathermy scratch pad is an effective tool for dermabrasion, as well as simultaneous debridement of burn wounds.

Under general anesthesia, the skin is cleansed with $10 \%$ povidone-iodine solution. To facilitate debridement, the self-adhesive of the diathermy scratch pad can be attached to the fingertips of the middle three fingers. As described above for chemical burns, using different combinations of angle, pressure, and speed, the surgeon can adjust the "angle of attack" on the foreign body tattooing, minimizing the tissue trauma need to remove each individual embedded foreign body. To simultaneously treat thermal burns, debridement with the diathermy scratch pad can be done with higher pressure and repetition, allowing debridement of even mid to deep dermal partial-thickness burns. The endpoint of the debridement is the complete re-

\section{Fig. 2. Patient 3 with acid burn}

Patient 3 sustained a 18\% total body surface area burn from exposure to methylacrylic acid. (A) A coagulum/eschar formed on the chest after exposure to the chemical, showing a distinct difference in coloration from the unaffected skin. (B) After debridement with the diathermy scratch pad, the burn wounds achieved $\mathrm{pH}$ neutralization, as indicated by the universal $\mathrm{pH}$ indicator strip.
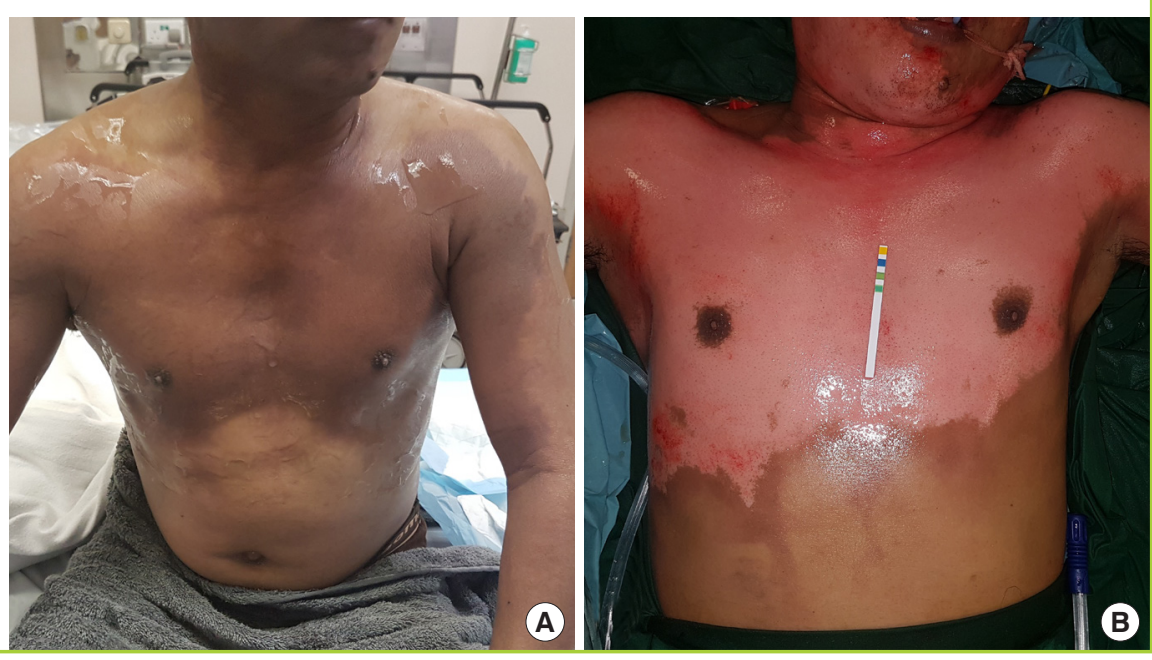


\section{Fig. 3. Patient 4 with acid burn}

Patient 4 sustained a 13\% total body surface area burn after exposure to sulfuric acid. (A) A dark brown eschar formed after exposure to the acid, clearly demarcating healthy and unhealthy skin. (B) After debriding the eschar, the true depth of the burn could be evaluated. As this was a deep dermal burn, it required further debridement and secondary skin grafting. (C) After 1 month, the patient had recovered well and returned to employment.
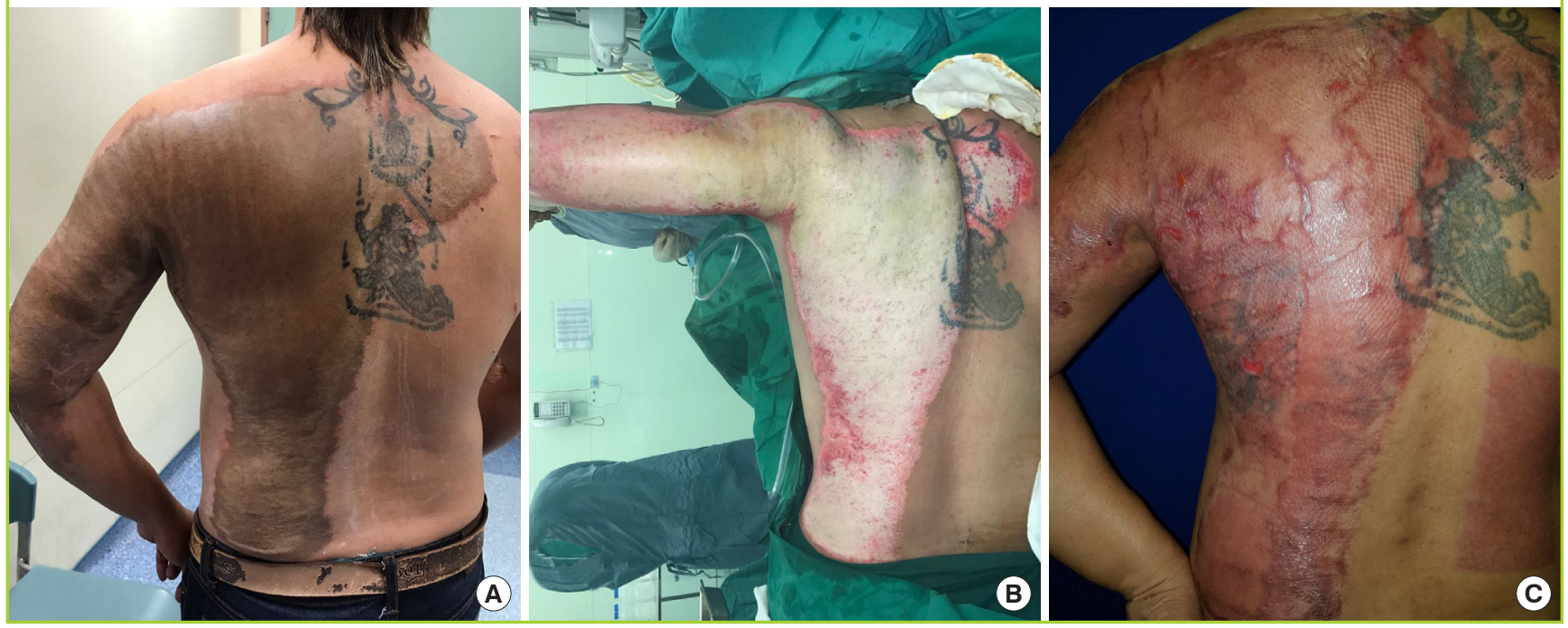

Table 1. Characteristics of the patient population

\begin{tabular}{|lclccccc|}
\hline Case & Age (yr) & Nature of burns & $\begin{array}{c}\text { Total body surface } \\
\text { area (\%) }\end{array}$ & $\begin{array}{c}\text { Foreign body } \\
\text { tattooing }\end{array}$ & $\begin{array}{c}\text { Length of stay } \\
\text { (day) }\end{array}$ & $\begin{array}{c}\text { Secondary skin } \\
\text { graft }\end{array}$ & $\begin{array}{c}\text { Length of } \\
\text { follow-up (mo) }\end{array}$ \\
\hline Patient 1 & 54 & Explosion & 13 & Yes & 7 & No & 13 \\
Patient 2 & 33 & Explosion & 2 & Yes & 3 & No & 12 \\
Patient 3 & 47 & Methacrylic acid & 18 & No & 10 & No & 6 \\
Patient 4 & 48 & Sulfuric acid & 13 & No & 28 & Yes & 12 \\
Patient 5 & 62 & Sodium hydroxide & 2.5 & No & 4 & No \\
\hline
\end{tabular}

moval of all foreign bodies and burnt skin. A healthy wound bed with healthy punctate bleeding can be achieved with this technique. Biobrane can now be applied as a wound dressing, with iodine gauzes as secondary dressing as per our protocol [3].

Between January and July 2017, a diathermy scratch pad was used on five patients for surgical debridement and dermabrasion (Table 1). The causes of burns included only chemical and explosion-related burns. The total body surface area involved ranged from $2 \%$ to $20 \%$. Eighty percent $(n=4)$ of the patients healed without further intervention. Twenty percent $(n=1)$ of the patients required a secondary skin graft due to secondary wound infection (Fig. 3). The average length of follow-up was 11.6 months.

\section{DISCUSSION}

Acid burns result in coagulative necrosis, caused by protein denaturation. This results in a layer of coagulum on the surface of the skin, which manifests as a thin eschar. This eschar can range from a light brown to a black color, and it can be very adherent and notoriously difficult to remove (Fig. 1). This layer of eschar is non-permeable and actually helps to limit further damage from the offending acid. Irrigation with saline or water to achieve neutralization is a universally accepted method. However, this layer of eschar is a non-permeable layer of contaminated skin, and neutralization is difficult to achieve as long as it remains present. Therefore, surgical debridement is recommended, but excision with a Goulian or Watson blade will imprecisely remove more tissue than needed. The Versajet hydrosurgery system offers a more refined method of tangential excision, but it is very expensive $[5,6]$. The authors believe that debridement with a diathermy scratch pad can achieve an even more precise method of debridement, whereby the offending tissue can be removed layer by layer via the principle of dermabrasion [7]. This ensures maximal preservation of healthy dermis, and in turn minimizes scarring [8]. Diathermy scratch pads are readily available in operating theatres and are already sterilized, facilitating their use in a sterile surgical field. The unit price for a dia- 
thermy scratch pad is less than US $\$ 2$, making it a very cheap tool compared to other methods.

Wounds caused by alkaline substances can appear superficial at first, but they often become full-thickness burns in 2 to 3 days. Unlike acids, alkalis can form soluble proteins upon binding with lipids and proteins, allowing easy passage of the hydroxyl ion into deeper tissue. Subsequently, a soft brownish gelatinous eschar is formed, as opposed to the thin hard eschar of an acid burn. To completely neutralize an alkali burn, the soft gelatinous eschar must be surgically debrided (Supplemental Video 1). In superficial alkali burns, the diathermy scratch pad is a very useful tool. Due to the higher propensity for burn conversion with alkali burns, the surgeon should perform a relook debridement with the option of skin grafting if necessary.

Foreign body tattooing is common in cases of explosion-related burns. The removal of the foreign bodies can be a time consuming and laborious task. Dermabrasion is an efficient method to treat large areas of foreign body tattooing. It can be performed using different methods, such as using sterile sandpaper or electrical dermabraders with a sterile diamond fraise [9]. Sterile sandpaper offers varying abrasive levels and is often not standardized [10]. The efficacy of electrical dermabraders is limited by the size and abrasiveness of the diamond fraise. The diathermy scratch pad addresses both disadvantages by having standardized abrasive levels and enabling dermabrasion to be performed over a larger surface area than is possible using a diamond fraise. It is also cheap and comes in a sterile pack. No additional electrical unit is necessary for its operation, and it can even be done in an outpatient setting.

In conclusion, the diathermy scratch pad is a cheap, sterile, and efficient tool that is especially suited to address stubborn eschars caused by chemical burns and large areas of foreign body tattooing.

\section{NOTES}

\section{Conflict of interest}

No potential conflict of interest relevant to this article was reported.

\section{Ethical approval}

The study was approved by the Institutional Review Board of Singapore General Hospital (IRB No. 2017/2444) and performed in accordance with the principles of the Declaration of Helsinki. Written informed consents were obtained.

\section{Patient consent}

The patients provided written informed consent for the publication and the use of their images.

\section{ORICD}

Allen Wei-Jiat Wong https://orcid.org/0000-0001-7924-5155

Qi En Hong https://orcid.org/0000-0002-2125-1031

Cheryl Li Yu Hui https://orcid.org/0000-0003-4207-3294

\section{Supplementary Material}

Supplemental Video 1. Fine debridement. This video demonstrates the precise fine debridement technique using the index finger in patient 5 .

Supplemental data can be found at:

https://doi.org/10.5999/aps.2018.00507.v001

\section{REFERENCES}

1. Chong SJ, Song C, Tan TW, et al. Multi-variate analysis of burns patients in the Singapore General Hospital Burns Centre (2003-2005). Burns 2009;35:215-20.

2. Hwee J, Song C, Tan KC, et al. The trends of burns epidemiology in a tropical regional burns centre. Burns 2016;42: 682-6.

3. Tay KS, Chong SJ, Tan BK. Impact of a newly implemented burn protocol on surgically managed partial thickness burns at a specialized burns center in Singapore. Ann Plast Surg 2016;76:276-9.

4. Chong SJ, Kok YO, Choke A, et al. Comparison of four measures in reducing length of stay in burns: an Asian centre's evolved multimodal burns protocol. Burns 2017;43: 1348-55.

5. Boudana DA, Wolber A, De Broucker V, et al. The use of Versajet hydrosurgery system in the treatment of vesicant burn caused by sulphur mustard: a propos of one case. Burns 2010;36:e44-8.

6. Klein MB, Hunter S, Heimbach DM, et al. The Versajet water dissector: a new tool for tangential excision. J Burn Care Rehabil 2005;26:483-7.

7. Seoighe DM, Power K. Use of the scratch pad for granulation tissue debridement. J Plast Reconstr Aesthet Surg 2010;63:e587.

8. Ogawa R. Keloid and hypertrophic scars are the result of chronic inflammation in the reticular dermis. Int J Mol Sci 2017;18:E606.

9. Campbell TM, Eisen DB. Electrobrasion: an alternative to dermabrasion. Dermatol Surg 2010;36:1739-42.

10. Kidwell MJ, Arpey CJ, Messingham MJ. A comparison of histologic effectiveness and ultrastructural properties of the electrocautery scratch pad to sandpaper for manual dermabrasion. Dermatol Surg 2008;34:1194-9. 